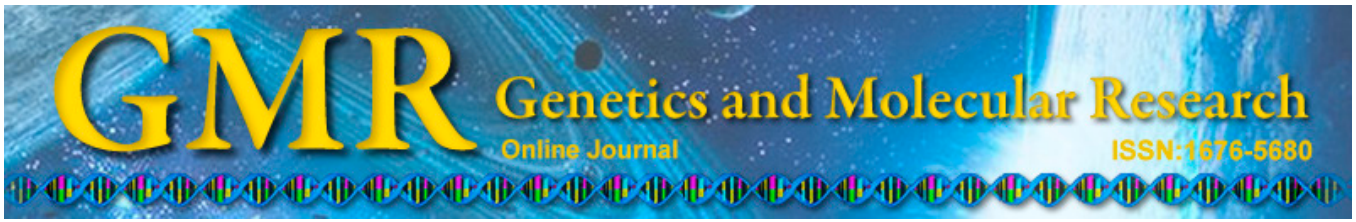

\title{
Acanthopanax senticosus attenuates inflammation in lipopolysaccharide-induced acute lung injury by inhibiting the NF-кB pathway
}

\author{
X.J. Fei ${ }^{1 *}$, L.L. Zhu ${ }^{2 *}$, L.M. Xia ${ }^{3}$, W.B. Peng ${ }^{4}$ and Q. Wang ${ }^{1}$ \\ ${ }^{1}$ Department of Hospital Infections, Shuguang Hospital Affiliated with \\ Shanghai University of Traditional Chinese Medicine, Shanhai, China \\ ${ }^{2}$ Department of Internal Medicine, Shuguang Hospital Affiliated with \\ Shanghai University of Traditional Chinese Medicine, Shanghai, China \\ ${ }^{3}$ Department of Internal Medicine, \\ Shanghai Municipal Hospital of Traditional Chinese Medicine, \\ Shanghai, China \\ ${ }^{4}$ Department of Traditional Chinese Medicine, \\ Shanghai Sixth People's Hospital Affiliated to Shanghai Jiao Tong University, \\ Shanghai, China \\ *These authors contributed equally to this study. \\ Corresponding author: Q. Wang \\ E-mail: wangqianshuguang@126.com \\ Genet. Mol. Res. 13 (4): 10537-10544 (2014) \\ Received November 21, 2013 \\ Accepted May 1, 2014 \\ Published December 12, 2014 \\ DOI http://dx.doi.org/10.4238/2014.December.12.16
}

\begin{abstract}
The dried roots of the plant Acanthopanax senticosus (AS) are used in traditional Oriental medicine and reportedly possess anti-inflammatory properties in vitro. However, whether AS has the same anti-inflammatory effect in vivo and the underlying mechanisms of this action remain unknown. In this study, we pretreated a mouse model of lipopolysaccharide-induced acute lung injury with AS and found that the levels of tumor necrosis factor (TNF)- $\alpha$, interleukin
\end{abstract}


(IL)-6, and neutrophils in bronchoalveolar lavage fluid were reduced and that inflammation in lung tissues was attenuated. To determine the mechanisms of these actions, we next assessed the effects of AS on the activation of the nuclear factor (NF)- $\kappa B$ pathway. We found that AS decreased the level of NF- $\mathrm{kB}$ and the DNA-binding activity of NF-kB. In summary, we found that AS attenuated the levels of TNF- $\alpha$ and IL-6 in the lung tissue of a mouse model of acute lung injury by inhibiting the NF- $\kappa \mathrm{B}$ pathway.

Key words: Acanthopanax senticosus; Inflammation; Interleukin-6; Nuclear factor- $\kappa B$; Tumor necrosis factor- $\alpha$

\section{INTRODUCTION}

Acanthopanax senticosus (AS), also known as ciwujia in the Chinese language, is a traditional Chinese herb widely used for treating a variety of diseases including hepatitis, gastric ulcers, ischemic heart disease, hypertension, and rheumatism (Lin and Huang, 2000; Gaffney et al., 2001; Yi et al., 2001; Hu et al., 2013). Previous studies found that AS suppressed proinflammatory factors such as interleukin (IL)-6 and tumor necrosis factor (TNF)- $\alpha$ in SW982 human synovial sarcoma cells (Yamazaki et al., 2007); however, whether AS has the same anti-inflammatory effect in vivo and the underlying mechanisms of this action remain unknown.

The nuclear factor (NF)- $\mathrm{kB}$ family contains 5 members: RelA (also known as p65), RelB, c-Rel, p105/p50, and p100/p52, which form both homodimers and heterodimers (Jeong et al., 2006). NF- $\mathrm{\kappa B}$ is a transcription factor that recognizes a common consensus DNA sequence and regulates large numbers of target genes, particularly genes involved in inflammation, injury, and stress (Tak and Firestein, 2001). IL-6 and TNF- $\alpha$ are cytokines that play essential roles in inflammation. NF- $\mathrm{kB}$ is typically localized to the cytoplasm as a p65 and p50 heterodimer. Activated NF- $\kappa B$ enters the nucleus and can promote the transcription of its target genes, including pro-inflammatory cytokine IL-6 and TNF- $\alpha$ (Snyder et al., 2002; He et al., 2006; Kim et al., 2009).

Recent studies found that AS suppressed not only the production of reactive oxygen species and nitric oxide in murine macrophages, but also the expression of inducible nitric oxide synthase by inhibiting intracellular peroxide production in lipopolysaccharide (LPS) plus interferon-gamma-stimulated RAW 264.7 cells (Lin et al., 2008). Moreover, NF- $\kappa B$ are transcription factors that mediate immune and inflammatory responses by promoting the expression of pro-inflammatory mediators such as inducible nitric oxide synthase, reactive oxygen species, and pro-inflammatory cytokines (Surh et al., 2008). Therefore, in the present study, we examined the anti-inflammatory effect of AS in LPS-induced acute lung injury (ALI) and the mechanisms of AS based on the reduction of NF- $\kappa \mathrm{B}$ signaling pathways.

\section{MATERIAL AND METHODS}

\section{Animals}

Forty-eight C57/BL6 male mice weighing $20 \pm 2 \mathrm{~g}$ were obtained from the Shanghai Shuguang Hospital, which is affiliated to the Shanghai University of Traditional Chinese 
Medicine SPF Animal Center (Shanghai, China). Mice were housed at $23^{\circ} \mathrm{C}$ with a 12 -h lightdark cycle and allowed free access to food and water. All animal experiments were approved by the Animal Care Committee of Shanghai University of Traditional Chinese Medicine University.

Mice were randomly divided into 4 groups $(\mathrm{N}=12$ in each group): control group, LPS group, AS group, and LPS+AS group. AS was purchased from Wusuli River Pharmacy Company (Hulin, Heilongjiang Province, China). Doses of AS $(20 \mathrm{mg} / \mathrm{kg})$ were determined based on results of our previous study (Zhao, 2008). In the AS group, doses were administered by tail vein injection. In the LPS+AS group, doses were administered by tail vein injection 1 $\mathrm{h}$ before LPS administration.

\section{Murine model of LPS-induced ALI}

The general procedure was modified from that described in previous experiments (Liao et al., 2012). Mice were anesthetized by an intraperitoneal injection of $0.1 \mathrm{~mL} 50 \mathrm{mg} / \mathrm{kg}$ pentobarbital sodium (Sigma, St. Louis, MO, USA) and placed in a supine position with the head placed on a board tilted at $45^{\circ}$. A midline incision was made in the neck and the trachea was exposed. LPS (Sigma) was dissolved in $0.9 \%$ saline at concentrations of $2 \mathrm{mg} / \mathrm{mL}$ and applied to the trachea at a dosage of $5 \mathrm{mg} / \mathrm{kg}$ using a 29-gauge needle. After intratracheal application, the mice were placed in a vertical position and rotated for $0.5-1 \mathrm{~min}$ to distribute the instillation evenly within the lungs.

\section{Bronchoalveolar lavage fluid (BALF) analysis and cell counts}

Mice were exsanguinated via the abdominal aorta, the trachea was cannulated, and the chest cavity was opened via a midline incision. The left main-stem bronchus was ligated, and the right lung was washed 3 times with $0.5 \mathrm{~mL}$ ice-cold sterile saline. In all cases, more than $95 \%$ of the total lavage volume $(1.5 \mathrm{~mL})$ was recovered. A $0.5-\mathrm{mL}$ aliquot of BALF was used to determine total cell and differential cell counts. The remaining BALF was centrifuged at $1000 \mathrm{~g}$ for $5 \mathrm{~min}$ at $4^{\circ} \mathrm{C}$ and the cell-free supernatant was stored at $-80^{\circ} \mathrm{C}$ for analysis of cytokines using an enzyme-linked immunosorbent assay (ELISA). The $0.5-\mathrm{mL}$ aliquot of BALF was subjected to hypotonic shock to lyse the red blood cells, and then total cell counts were determined using a hemacytometer. Cells were adjusted to a concentration of $5 \times 10^{5} / \mathrm{mL}$ in phosphate-buffered saline. Cells were stained with Wright's stain. Differential cell counts were conducted for samples of 200 cells.

\section{Nuclear extraction and Western blot}

Lung tissue samples were collected $6 \mathrm{~h}$ after LPS treatment. A nuclear extraction kit (Ab110168; Abcam, Cambridge, UK) was used to prepare nuclear extracts from lung tissue. For Western blotting, frozen lung tissues were homogenized and the lysates were prepared in ice-cold lysis buffer. Nuclear extracts or total protein were collected and normalized for equal amounts of total protein measured using the bicinchoninic acid method. Next, $70 \mu \mathrm{g}$ protein from each sample was separated on a sodium dodecyl sulfate-polyacrylamide gel and transferred to polyvinylidene fluoride membranes. The membranes were blocked with $5 \%$ nonfat milk and incubated overnight with primary anti-NF-kB antibody (1:1000; sc-109; Santa Cruz Biotechnology, Santa Cruz, CA, USA), anti-IL-6 antibody (1:1000; ab6672; Abcam), anti- 
TNF- $\alpha$ antibody (1:1000; AB1837P; Millipore, Billerica, MA, USA), and anti- $\beta$-actin (1:5000; Sigma) at $4^{\circ} \mathrm{C}$, followed by incubation with the corresponding horseradish peroxidase-conjugated secondary antibody for $4 \mathrm{~h}$. $\beta$-actin protein was immunodetected as an internal standard.

\section{ELISA}

IL-6 and TNF- $\alpha$ protein levels in the sciatic nerve were measured using ELISA kits according to manufacturer instructions (R\&D Systems, Minneapolis, MN, USA).

\section{Electrophoretic-mobility shift assay (EMSA)}

An EMSA kit (GS-0030; Signosis, Santa Clara, CA, USA) was used to assess the transcription-binding activity of NF- $\mathrm{kB}$ as described previously (Zhang et al., 2013). Next, $5 \mathrm{mg}$ nuclear extract was incubated with $1 \mu \mathrm{L}$ poly d(I-C), $2.0 \mu \mathrm{L} 5 \mathrm{X}$ binding buffer, and 1.0 $\mu \mathrm{L}$ transcription factor probe in a $0.5-\mathrm{mL}$ microcentrifuge tube at $20^{\circ}-23^{\circ} \mathrm{C}$ for $30 \mathrm{~min}$ in a polymerase chain reaction machine. We added $1.0 \mu \mathrm{L}$ cold transcription factor probe to this reaction as the cold probe control. Samples were then loaded onto a $6.5 \%$ non-denaturing polyacrylamide gel, which was run at $100 \mathrm{~V}$ and transferred to $60 \mathrm{~V}$ for $1 \mathrm{~h}$ at $4^{\circ} \mathrm{C}$. The membrane was imaged using a chemiluminescence imaging system (Bio-Rad, Hercules, CA, USA).

\section{Statistical analysis}

Data are reported as means $\pm \mathrm{SD}$. The Student $t$-test and two-way analysis of variance were used to compare differences from the control group. $\mathrm{P}<0.05$ was considered to be statistically significant. Significance tests were two-tailed. A normality test showed that the data were normally distributed. The GraphPad software Prism 5 (San Diego, CA, USA) was used for data analysis.

\section{RESULTS}

\section{AS reduced the number of leukocytes in BALF in the mouse model of LPS-induced ALI}

In the mouse model of LPS-induced ALI, we pretreated the mice $1 \mathrm{~h}$ before LPS administration. Total cell counts in BALF increased by 6,12 , and $24 \mathrm{~h}$ (Figure 1), and differential cell counts revealed that the increase in leukocytes was mainly attributable to neutrophils (Table 1). In contrast, the numbers of macrophages and lymphocytes did not differ significantly between groups. At each time point, AS significantly reduced the number of leukocytes, mainly neutrophils, in BALF.

\section{AS reduced the levels of TNF- $\alpha$ and IL-6 in BALF in the mouse model of LPS-induced ALI}

Protein levels of both TNF- $\alpha$ and IL-6 in BALF were significantly increased after LPS administration and attenuated by AS pretreatment (Figure 2A and B). The concentrations of both TNF- $\alpha$ and IL-6 in BALF were also significantly increased after LPS administration and attenuated by AS pretreatment according to the ELISA results (Figure $2 \mathrm{C}$ and D). 


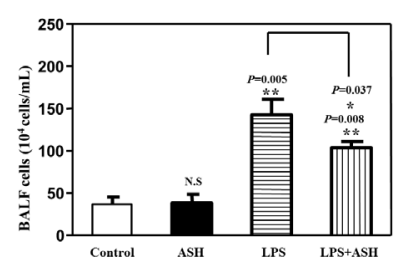

B

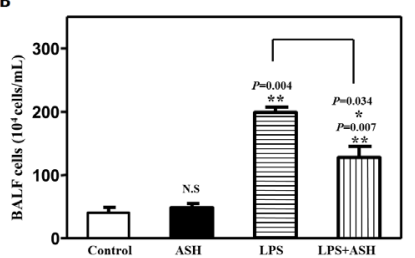

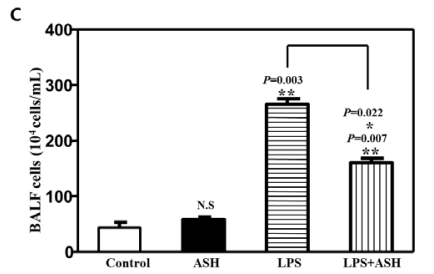

Figure 1. AS reduced the number of leukocytes in BALF in the mouse model of LPS-induced ALI. BALF samples were collected at 6 (A), 12 (B), and $24 \mathrm{~h}(\mathbf{C})$ after LPS administration. LPS increased the numbers of leukocytes in BALF of mice, an effect that was partially reversed by AS. Values are reported as means $\pm \mathrm{SD}(\mathrm{N}=3) . * \mathrm{P}<0.05$, $* * \mathrm{P}<0.01$. LPS $=$ lipopolysaccharide; $\mathrm{AS}=$ Acanthopanax senticosus.

Table 1. Differential cell counts (expressed as cells x $10^{4}$ ) in BALF from mice subjected to LPS-induced ALI following different treatments.

\begin{tabular}{|c|c|c|c|c|c|c|c|c|c|}
\hline \multirow[t]{3}{*}{ Group } & \multicolumn{9}{|c|}{ Time after LPS induction of ALI } \\
\hline & \multicolumn{3}{|c|}{$6 \mathrm{~h}$} & \multicolumn{3}{|c|}{$12 \mathrm{~h}$} & \multicolumn{3}{|c|}{$24 \mathrm{~h}$} \\
\hline & Macrophages & Neutrophils & Lymphocytes & Macrophages & Neutrophils & Lymphocytes & Macrophages & Neutrophils & Lymphocytes \\
\hline Control & $14.23 \pm 5.45$ & $0.03 \pm 0.02$ & $0.62 \pm 0.32$ & $17.65 \pm 3.21$ & $0.04 \pm 0.01$ & $0.52 \pm 0.18$ & $23.56 \pm 4.61$ & $0.04 \pm 0.02$ & $0.57 \pm 0.22$ \\
\hline AS & $19.33 \pm 7.28$ & $0.08 \pm 0.03$ & $0.73 \pm 0.22$ & $22.43 \pm 4.37$ & $0.15 \pm 0.04$ & $1.28 \pm 0.72$ & $21.78 \pm 5.24$ & $0.24 \pm 0.06$ & $1.21 \pm 0.67$ \\
\hline LPS & $15.15 \pm 4.33$ & $110.45 \pm 5.46^{*}$ & $2.56 \pm 0.23$ & $15.26 \pm 3.87$ & $153.45 \pm 6.76^{*}$ & $3.42 \pm 0.59$ & $18.99 \pm 4.23$ & $197.22 \pm 5.26^{*}$ & $3.66 \pm 0.45$ \\
\hline LPS+AS & $17.86 \pm 2.89$ & $85.57 \pm 6.91^{* \#}$ & $1.65 \pm 0.48$ & $17.33 \pm 3.19$ & $104.65 \pm 7.82^{\text {*\# }}$ & $2.76 \pm 0.88$ & $18.34 \pm 5.38$ & $136.34 \pm 5.38^{* \#}$ & $2.43 \pm 0.78$ \\
\hline
\end{tabular}

Data are reported means $\pm \mathrm{SD}, \mathrm{N}=3 . \mathrm{BALF}=$ bronchoalveolar lavage fluid; $\mathrm{AS}=$ Acanthopanax senticosus. $* \mathrm{P}<$ 0.05 compared with the LPS group at each time point. ${ }^{*} \mathrm{P}<0.05$ compared with the AS+LPS group at each time point.

A

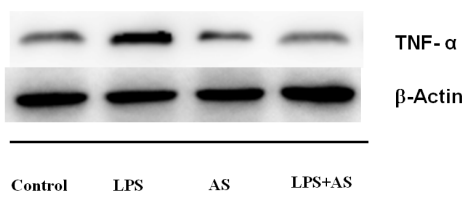

C

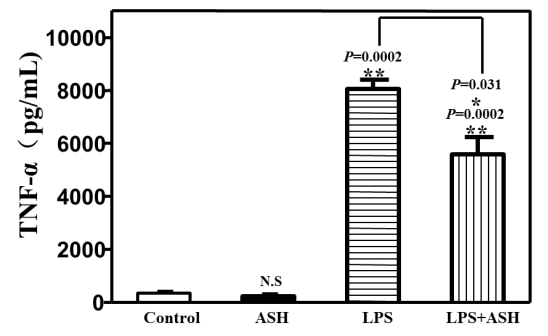

B

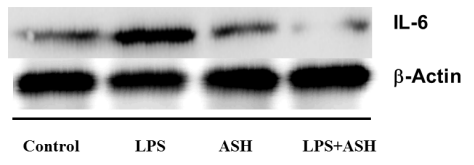

D

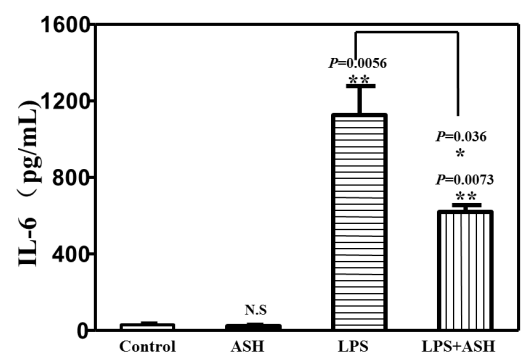

Figure 2. AS reduced the levels of TNF- $\alpha$ and IL-6 in BALF in the mouse model of LPS-induced ALI. A. LPS increased the protein levels of TNF- $\alpha$, an effect attenuated by AS. B. LPS increased the protein levels of IL-6, which was attenuated by AS. C. LPS increased the concentrations of TNF- $\alpha$; AS attenuated the increase based on ELISA results. D. LPS increased the concentrations of IL-6, which was attenuated by AS according to ELISA results. Values for Western blotting are reported means $\pm \mathrm{SD}(\mathrm{N}=3) . * \mathrm{P}<0.05, * * \mathrm{P}<0.01$. LPS $=$ lipopolysaccharide; AS = Acanthopanax senticosus. 


\section{AS attenuated the levels of TNF- $\alpha$ and IL-6 in lung tissue in the mouse model of LPS-induced ALI by inhibiting the NF- $\kappa$ B pathway}

Western blotting of NF- $\mathrm{kB}$ showed that AS reduced the nuclear levels of NF- $\kappa \mathrm{B}$ in the mouse model of LPS-induced ALI compared with the LPS group, but did not alter the total levels (Figure 3A). EMSA of NF- $\kappa$ B showed that AS inhibited the transcription binding activity of NF-kB in the mouse model of LPS-induced ALI compared with the LPS group (Figure $3 \mathrm{~B})$. NF- $\mathrm{\kappa B}$ is a transcription factor that regulates the expression levels of TNF- $\alpha$ and IL-6; thus, these results suggest that AS attenuated the levels of TNF- $\alpha$ and IL-6 in lung tissue in the mouse model of LPS-induced ALI by inhibiting the NF- $\mathrm{KB}$ pathway.

A

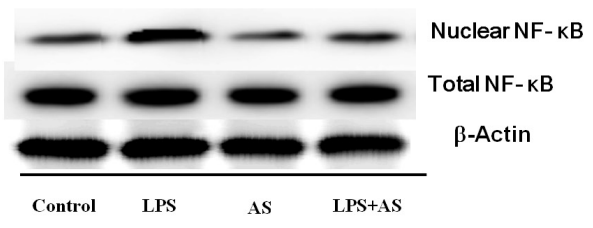

B

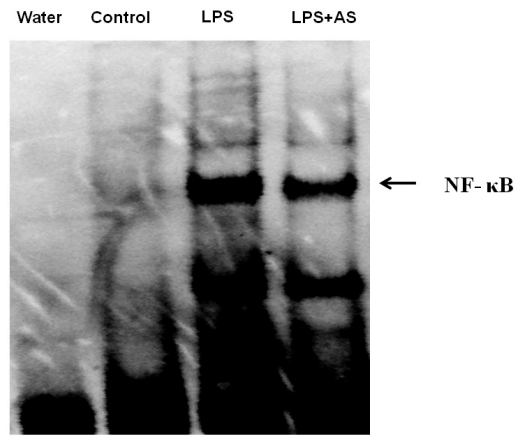

Figure 3. AS reduced nuclear levels of NF- $\mathrm{kB}$ and the DNA-binding activity of NF-kB in the lung tissue of the mouse model of LPS-induced ALI. A. LPS increased the nuclear levels of NF- $\kappa B$, attenuated by AS. B. LPS increased the DNA-binding activity of NF-kB, and AS attenuated the increase. Values of Western blotting are reported means \pm SD $(\mathrm{N}=3) . * \mathrm{P}<0.05, * * \mathrm{P}<0.01 . \mathrm{LPS}=$ lipopolysaccharide; $\mathrm{AS}=$ Acanthopanax senticosus.

\section{DISCUSSION}

Increasing attention has been given to the use and mechanism of action of phytochemicals in clinical settings. Phytochemicals are natural substances that can be extracted from plants. AS, a widely used traditional Chinese herb in the clinical setting, is considered to be a potential antioxidant and anti-inflammatory agent (Fujikawa et al., 2005). Although many studies have been conducted to investigate the anti-inflammatory properties of AS, the mechanisms of its inflammatory responses are unknown. In the current study, we explored the potential of AS to be used as a therapeutic agent against inflammation in clinical settings and the underlying mechanism of this effect. We employed a model of ALI induced by LPS to assess the effects of AS on the levels of IL- 6 and TNF- $\alpha$ as well as the activation of NF- $\kappa$ B. The results of this study indicate that AS exerts its anti-inflammatory effects by inhibiting NF- $\kappa B$ signaling.

In this study, we pretreated the mouse model of LPS-induced ALI with AS and found that the levels of TNF- $\alpha$, IL- 6 , and neutrophils in BALF were reduced and that inflammation in lung tissues was attenuated. Normally, in inflammatory diseases, neutrophils are rapidly recruited to sites of inflammation; there, they kill invading bacteria through phagocytosis involving the release of preformed granular enzymes and proteins, as well as producing a range 
of oxygen species (Serhan, 2010). However, the highly destructive activity of neutrophils can also pose a risk to healthy tissues. In our mouse model of LPS-induced ALI, AS at a dose of 20 $\mathrm{mg} / \mathrm{kg}$ significantly and rapidly reduced the numbers of neutrophils in BALF and attenuated inflammation and ALI scores in lung tissues.

Proinflammatory cytokines play a critical role in ALI; persistently elevated levels of proinflammatory cytokines such as TNF- $\alpha$ and IL-6 have been associated with worse outcome in patients with ALI (Calder, 1997; Minamino and Komuro, 2006). Because TNF- $\alpha$ and IL-6 are considered to be markers of inflammation, their increased levels after LPS administration in our mouse model of ALI suggest that our model is valid. In this study, we pretreated the mouse model of LPS-induced ALI with AS and we found that AS down-regulated levels of TNF- $\alpha$ and IL-6 in BALF from mice with LPS-induced ALI. In summary, we suggest that the ability of AS to reduce neutrophil counts at inflammatory sites and to attenuate the levels of TNF- $\alpha$ and IL-6 in lung tissue in our mouse model of ALI involves a combination of antiinflammatory and pro-resolving mechanisms.

Future studies will assess the effects of AS on activation of the NF- $\mathrm{BB}$ pathway. NF$\kappa \mathrm{B}$ is a transcription factor that recognizes a common consensus DNA sequence and regulates large numbers of target genes, particularly the genes involved in inflammation, injury, and stress (Murakami et al., 2011). IL-6 and TNF- $\alpha$ are cytokines that play essential roles in the inflammation process. In this study, we also found that AS decreased the level of NF-kB and the DNA-binding activity of NF-kB.

Our results strongly suggest that AS attenuated the levels of TNF- $\alpha$ and IL-6 in lung tissue in a mouse model of ALI by inhibiting the NF-kB pathway.

\section{REFERENCES}

Calder PC (1997). n-3 polyunsaturated fatty acids and cytokine production in health and disease. Ann. Nutr. Metab. 41: 203-234.

Fujikawa TKN, Shimada A, Ogata M, Suzuki I, et al. (2005). Effect of sesamin in Acanthopanax senticosus HARMS on behavioral dysfunction in rotenone-induced parkinsonian rats. Biol. Pharm. Bull. 28: 169-172.

Gaffney BT, Hügel, HM and Rich PA (2001). Panax ginseng and Eleutherococcus senticosus may exaggerate an already existing biphasic response to stress via inhibition of enzymes which limit the binding of stress hormones to their receptors. Med. Hypotheses 56: 567-572.

He HJ, Zhu TN, Xie Y, Fan J, et al. (2006). Pyrrolidine dithiocarbamate inhibits interleukin-6 signaling through impaired STAT3 activation and association with transcriptional coactivators in hepatocytes. J. Biol. Chem. 281: 31369-31379.

Hu W, Yeo JH, Jiang Y, Heo SI, et al. (2013). The antidiabetic effects of an herbal formula composed of Alnus hirsuta, Rosa davurica, Acanthopanax senticosus and Panax schinseng in the streptozotocin-induced diabetic rats. Nutr. Res. Pract. 7: 103-108.

Jeong WS, Jun M and Kong AN (2006). Nrf2: a potential molecular target for cancer chemoprevention by natural compounds. Antioxid. Redox Signal. 8: 99-106.

Kim KS, Oh da H, Choi HM, Bang JS, et al. (2009). Pyrrolidine dithiocarbamate, a NF-kappaB inhibitor, upregulates MMP-1 and MMP-13 in IL-1beta-stimulated rheumatoid arthritis fibroblast-like synoviocytes. Eur. J. Pharmacol. 613: $167-165$.

Liao Z, Dong J, Wu W, Yang T, et al. (2012). Resolvin D1 attenuates inflammation in lipopolysaccharide-induced acute lung injury through a process involving the PPAR $\gamma / \mathrm{NF}-\mathrm{\kappa B}$ pathway. Respir. Res. 2: 110.

Lin CC and Huang PC (2000). Antioxidant and hepatoprotective effects of Acathopanax senticosus. Phytother. Res. 14: 489-494.

Lin QY, Jin LJ, Cao ZH and Xu YP (2008). Inhibition of inducible nitric oxide synthase by Acanthopanax senticosus extract in RAW264.7 macrophages. J. Ethnopharmacol. 118: 231-236.

Minamino T and Komuro I (2006). Regeneration of the endothelium as a novel therapeutic strategy for acute lung injury. J. Clin. Invest. 116: 2316-2319. 
Murakami T, Suzuki K, Tamura H and Nagaoka I (2011). Suppressive action of resolvin D1 on the production and release of septic mediators in D-galactosamine-sensitized endotoxin shock mice. Exp. Ther. Med. 2: 57-61.

Serhan CN (2010). Novel lipid mediators and resolution mechanisms in acute inflammation: to resolve or not? Am. J. Pathol. 177: 576-591.

Snyder JG, Prewitt R, Campsen J and Britt LD (2002). PDTC and Mg132, inhibitors of NF-kappaB, block endotoxin induced vasodilation of isolated rat skeletal muscle arterioles. Shock 17: 304-307.

Surh YJ, Kundu JK and Na HK (2008). Nrf2 as a master redox switch in turning on the cellular signaling involved in the induction of cytoprotective genes by some chemopreventive phytochemicals. Planta Med. 74: 1526-1539.

Tak PP and Firestein GS (2001). NF-kappaB: a key role in inflammatory diseases. J. Clin. Invest. 107: 7-11.

Yamazaki T, Shimosaka S, Sasaki H, Matsumura T, et al. (2007). (+)-Syringaresinol-di-O-beta-D-glucoside, a phenolic compound from Acanthopanax senticosus Harms, suppresses proinflammatory mediators in SW982 human synovial sarcoma cells by inhibiting activating protein-1 and/or nuclear factor-kappaB activities. Toxicol. In Vitro 21: 1530-1537.

Yi JM, Kim MS, Seo SW, Lee KN, et al. (2001). Acanthopanax senticosus root inhibits mast cell-dependent anaphylaxis. Clin. Chim. Acta 312: 163-168.

Zhang L, Zhang J, Yang L, Dong Y, et al. (2013). Isoflurane and sevoflurane increase interleukin-6 levels through the nuclear factor-kappa B pathway in neuroglioma cells. Br J Anaesth. (Suppl 1): i82-91.

Zhao XY, Li Y, Yu XF, et al. (2008). Protective effect of compound Acanthopanax senticosus injection on myocardial ischemia-reperfusion injury in rats and its mechanism. Chin. J. Gerontol. 6: 23-25. 